\title{
Significant induction of embryonic stem (ES) cells differentiating into functional hepatocytes
}

Fuming $\mathrm{Li}^{1,2}$, Chenhuan $\mathrm{Xu}^{1,2}$, Yangfang $\mathrm{Li}^{3}$, Xiaojing $\mathrm{Pan}^{4}$, Xiaowen $\mathrm{Lv}^{1,2}$, Xiaoyan Ding ${ }^{1,2}$, Yiping $\mathrm{Hu}^{3}, \mathrm{Xin}$ Wang $^{1,2,4}$

${ }^{1}$ The key laboratory of Molecular Cell Biology, ${ }^{2}$ The key laboratory of Stem Cell Biology Shanghai, Institute of Biochemistry and Cell Biology, Shanghai Institute of Biological Sciences, Shanghai, China; ${ }^{3}$ Department of Cell Biology, Second Military Medical University, Shanghai, China, ${ }^{4}$ Stem Cell Institute, University of Minnesota, Minneapolis, USA

ES cells are pluripotent stem cells that can become many differentiated cells both in vivo and in vitro. It have been indicated that mouse ES cells could differentiate into functional hepatocytes under several types of treatments in cultures and after transplantations. These previous publications encouraged us to use human ES cell to derive functional human hepatocytes for potential clinical therapy in future. For this long-term goal, it is necessary to improve the efficiency of hepatic differentiation from ES cells. Different from the natural process of differentiation for embryonic cells during embryogenesis, differentiation of ES cells in vitro is an artificial process, which provides us with the chance to derive hepatocytes efficiently by the optimal design of selecting medium contents and activating of signaling pathways. We have successfully divided the whole process of ES cells differentiating functional hepatocytes into multi-steps. Recently, our results indicated that ES cells cultured in monolayer could be directly induced to differentiate into definitive endodermal cells. The components required for maintaining the survival and differentiation of ES cells under monolayer culture have already been determined. Activation of Wnt signally pathway is necessary for the differentiation of definitive endodermal cells from ES cells. Therefore, the agonist to activate Wnt signaling pathway was used to enhance the production of ES cell-derived definitive endodermal (ESDE) cells. We have found that a combined activation of both Wnt signally pathway and Nodal signally pathway resulted in a production of $80 \%$ to $90 \%$ ESDE cells in total ES cell-derived cells. For the next step, the self-renewal of ESDE cells maintaining in culture will be tested. ESDE cells are expected for further differentiation into hepatoblasts, which are bi-potential hepatic progenitor cells with expressions of both albumin and cytokeratin-19. The hepatoblasts could become functional hepatocytes after transplantation into liver of mouse with chronic liver injury. In conclusion, ES cells cultured in monolayer could be directly induced to differentiate into definitive endodermal cells. The activations of both Wnt pathway and Nodal pathway have been found to be better than single activation of Nodal pathway during the process of ES cells differentiating into ESDE cells. The newly established protocol in our study will significantly improve the efficiency of induced hepatic differentiation from ES cells.

Keywords: embryonic stem (ES) cells, hepatocytes, signaling pathway

Cell Research (2008) 18:s87. doi: 10.1038/cr.2008.177; published online 4 August 2008

Correspondence: Xin Wang

E-mail: wangx@sibs.ac.cn

Xin Wang $\mathrm{PhD}$, is the co-director of Key laboratory of Stem cell Biology, China Academy of Science in Shanghai. Dr. Wang received a $\mathrm{PhD}$ in Molecular Pharmacology from State University of New York at
Buffalo. He is current Professor/Principle Investigator in Shanghai Institute of Biological Sciences (SIBS), the associate director of Key laboratory of Molecular Cell Biology in Shanghai Institute of Biochemistry and Cell Biology, SIBS. He is a fellow of AAAS. Dr. Wang's research focuses on embryonic stem (ES) cell-derived hepatic cells. Please his lab website for more details. http://www.sibcb.ac.cn/PI.asp?id=23 